institut D'histologie Et D'embryologie - école de Médecine GEN ÈVE

\title{
Athrocytose et transfert des colorants colloïdaux et du pigment chez l'embryon et le têtard de Grenouille
}

par

\section{Anne-Marie DU BOIS}

Avec 6 figures dans le texte.

La Grenouille a déjà été utilisée, comme animal d'expériences, dans de nombreuses recherches sur l'athrocythose et l'élimination des colorants colloïdaux, expérimentalement introduits dans l'organisme. Cependant si ces faits sont bien connus chez l'adulte, ils n'ont pas encore été systématiquement étudiés au cours de l'ontogénèse. Il nous a paru intéressant de reprendre la question dans son ensemble, en examinant successivement tous les stades du développement de la neurula à la jeune Grenouille venant de se métamorphoser; nous nous proposons dans ce travail de préciser le moment où les éléments du système réticulo-endothélial sont assez différenciés pour pouvoir commencer à fonctionner comme athrocytes et par quelles voies l'organisme en formation est capable d'éliminer les colloïdes d'origine exogène qui l'encombrent.

Au cours de ces recherches, nous avons constaté que le transfert et l'élimination de l'énorme surcharge de pigment, que renferme la plupart des tissus embryonnaires, se font selon des mécanismes physiologiques fort comparables à ceux de l'athrocytose et de l'élimination des colloïdes d'origine exogène. A maints égards, le pigment de l'embryon de têtard de Grenouille se comporte comme un colloïde étranger à l'organisme, dont il faut que ce dernier se 
débarrasse. Ce problème du métabolisme du pigment nous a paru mériter une étude un peu détaillée.

Toutes les recherches expérimentales sur le mécanisme de la fonction histiocytaire ou athrocytose (colloïdopexie, Speicherung, storage sont des termes équivalents), tant chez les Mammifères que chez les Vertébrés inférieurs et chez les Invertébrés, ont été faites en introduisant dans l'organisme - par injection, par voie orale, etc. - des colloïdes facilement décelables, par la suite, dans les cellules qui les ont captés soit par leur coloration propre, soit par une réaction chimique simple. A la fin du siècle dernier et au commencement de celui-ci, la notion de colloïde étant encore fort mal connue, les expérimentateurs ont injecté au hasard des colorants dits "vitaux " et constaté que les uns étaient fixés par certaines cellules particulières tandis que les autres étaient rapidement éliminés. Au fur et à mesure que la notion de colloïde se précisait, on a constaté que les cellules du système réticulo-endothélial ne pouvaient capter que des colloïdes, d'où le nom de colloïdopexie attribué à cette fonction physiologique. Enfin, à la suite de nombreux travaux récents on est arrivé à resserrer encore davantage le problème et l'on considère actuellement que les athrocytes ne sont capables de fixer que les colloïdes acides et électro-négatifs (von Moellendorff 1918-1925). A côté de ces deux propriétés indispensables pour provoquer une réponse des cellules athrocytaires, il en existe une troisième, qui joue un rôle important, la grosseur des particules colloïdales. Les travaux de GÉrard et Cordier $(1932,1933)$ ont en particulier montré qu'il existe chez les Vertébrés et même chez les Invertébrés (Du Bors, 1942) une "athrocytose sélective ", les divers types d'athrocytes n'étant pas capables de fixer indifféremment les particules de n'importe quelle grosseur.

Dans la partie expérimentale de ce travail nous avons tenu compte de ce facteur en choisissant pour les injecter quatre colloïdes qui diffèrent essentiellement - pour le but qui nous intéresse - par la grosseur de leurs particules. Les diamètres de ces particules, calculés en angström ( $\AA=10^{-7} \mathrm{~mm}$.), ont été établis par Gérard et Cordier (1933). Ce sont: le bleu trypan, dont les particules extrêmement ténues ont un diamètre ne dépassant pas $6,5 \AA$, le sol de bleu trypan étant à la limite d'une solution vraie. Le saccharate de fer à particules de $24,6 \AA$ de diamètre, le bleu de 
Prusse pour injection à particules de $115,8 \AA$ et enfin l'encre de Chine pour injection de Grübler à très grosses particules, dont le diamètre n'a pas été très exactement déterminé, mais qui atteint presque la limite de visibilité.

\section{MATÉRIEL ET TECHNIQUE}

Nous avons utilisé dans nos expériences des embryons de Rana temporaria et des têtards de $R$. temporaria et $R$. esculenta. Les résultats obtenus sur les têtards de ces deux espèces ont été tout à fait identiques et les deux catégories d'expériences seront décrites simultanément. Chez les têtards immobilisés au M. S. 222, les différents colloïdes ont été injectés dans la cavité coelomienne à l'aide d'une micropipette ${ }^{1}$. L'endroit le plus indiqué pour faire la piqûre sans risque de léser les organes est la région dorso-latérale postérieure, en introduisant la pipette derrière la masse intestinale.

En piquant directement la paroi ventrale, on court le risque de blesser l'intestin ou de provoquer une éventration ultérieure, dès que le têtard se remet à nager.

Chez les embryons, l'injection a également été pratiquée dans la région dorso-latérale mais malgré toutes les précautions, le liquide injecté n'a que rarement été déposé exclusivement dans le coelome; la pipette pénètre toujours plus ou moins profondément dans l'épaisseur des grosses cellules endoblastiques surchargées de vitellus qui forment la paroi du tube digestif. Il a naturellement été nécessaire de débarrasser au préalable les embryons de leurs coques gélatineuses et de la membrane vitelline.

Pour les injections d'embryons, nous n'avons utilisé que le bleu trypan ou le bleu de Prusse aux dilutions de $1 \%$. Chez les têtards, nous avons injecté, outre ces deux bleus aux mêmes dilutions, du saccharate de fer à $5 \%$ et de l'encre de Chine de Grübler diluée au quart; les dilutions étaient faites avec de l'eau distillée stérilisée. Le saccharate de fer a été démasqué sur coupes, sous forme de bleu de Prusse, par la méthode de Turnbull; l'encre de Chine a nécessité une dépigmentation ultérieure assez poussée des coupes par la méthode de Kopsch.

1 En 1942, une partie des injections de têtards a été exécutée par M. Авоїм, de Lisbonne, qui désirait se mettre au courant de la technique. 
Il nous a été impossible d'évaluer avec précision la quantité de liquide injecté, le calibre et la longueur de la portion capillaire des pipettes n'étant jamais tout à fait identiques. Nous avons empiriquement injecté la quantité de colorant qui voulait bien pénétrer et comme la piqûre provoque, surtout chez l'embryon et les petits têtards, une grosse lésion par rapport à la taille de l'animal, une partie du colorant injecté s'est toujours écoulée par la plaie. Chez le têtard, l'injection massive de l'un des deux colorants bleus est immédiatement suivie de l'apparition d'une coloration bleue plus ou moins intense de tout le corps, coloration qui s'atténue au bout de quelques heures déjà, au fur et à mesure que le colorant est fixé par les athrocytes ou éliminé par voie rénale ou intestinale.

Pour disposer d'un matériel histologique suffisant, chaque série d'injections a comporté au moins une vingtaine d'individus, provenant tous de la même ponte et de tailles à peu près égales. En général nous avons injecté à la suite l'une de l'autre au moins deux séries d'animaux provenant de la même ponte, l'une au bleu trypan l'autre au bleu de Prusse.

Pour les embryons, les longueurs en $\mathrm{mm}$. indiquées dans le texte désignent la longueur totale de ceux-ci, de l'extrémité antérieure de la tête au bout de la queue. Pour les têtards après éclosion, les chiffres indiqués ne donnent que la longueur du corps, du bout du museau à la naissance de la queue. Chez le têtard en effet la longueur totale du museau à l'extrémité de la queue ne fournit pas une donnée exacte de la croissance de l'animal. La longueur totale maximale est atteinte avant même que les patte postérieures aient fait leur apparition et le corps continue ensuite à s'allonger jusqu'après la métamorphose alors que la queue régresse.

Les embryons ont été fixés 24 à 72 heures après l'injection, les têtards après 24 ou 48 heures. Au début, nous avons utilisé comme fixateur le formol acétifié à $5 \%$, puis le liquide de Bourn. Les larves et les petits têtards ont été coupés in toto, en coupes frontales. Chez les gros têtards nous avons supprimé la tête et la queue et coupé en section frontale tous les organes abdominaux in situ.

Dans les séries à l'encre de Chine ou au saccharate de fer, les coupes ont simplement été colorées au rouge pour noyau, après dépigmentation ou après réaction de Turnbull. 


\section{PREMIÈRE PARTIE}

\section{TRANSFERT ET ÉLIMINATION DU PIGMENT EMBRYONNAIRE}

Les embryons de $R$. temporaria sont caractérisés par leur richesse en pigment noir. La quantité de pigment varie suivant les tissus et semble être inversement proportionnelle à la quantité de plaquettes vitellines qu'ils renferment. Les cellules d'origine ectoblastique, cellules épidermiques et nerveuses, totalement dépourvues de vitellus, sont littéralement bourrées de fines granulations noires. Les cellules des tissus mésoblastiques, muscles, mésenchyme, sang, etc... renferment côte à côte des plaquettes vitellines et des granulations pigmentaires en quantités plus ou moins considérables. Enfin, les cellules endoblastiques de la paroi intestinale, caractérisées par leur énorme surcharge de plaquettes vitellines ne contiennent que très peu de granulations pigmentaires. $\mathrm{Au}$ cours du développement, au fur et à mesure de la résorption des plaquettes vitellines, on assiste à une élimination progressive du pigment dans les tissus.

Cette richesse en pigment des tissus ecto- et mésoblastiques doit vraisemblablement jouer un rôle important dans les processus de la régulation thermique et de la résistance à la luminosité ${ }^{1}$.

$\mathrm{Au}$ fur et à mesure de la différenciation des organes au cours de la vie embryonnaire, on assiste à un remaniement du pigment qui est progressivement éliminé de la plupart des tissus. La pigmentation noire intense, caractéristique des stades embryonnaires, commence à s'éclaircir peu avant l'éclosion, le processus se poursuit

${ }^{1}$ L'embryon de Grenouille, emprisonné dans une coque gélatineuse épaisse, immergé à faible profondeur dans une eau stagnante, est soumis à des variations extrêmes de température et de luminosité.

L'œuf de Triton cristatus, à coque gélatineuse beaucoup moins volumineuse, est accolé au moment de la ponte à la face inférieure d'une feuille immergée que la femelle replie ensuite autour de lui. Les conditions, d'échauffement et d'éclairage doivent être beaucoup moins variables; l'embryon est complètement dépourvu de pigment, celui-ci n'apparait que tardivement dans les tissus, peu avant l'éclosion de la larve. 
au cours de la vie larvaire si bien que le têtard prêt à se métamorphoser est devenu plus ou moins gris mordoré. Ce remaniement du pigment comporte deux phénomènes distincts et simultanés: d'une part l'élimination des granulations pigmentaires de la plupart des organes, d'autre part la différenciation de mélanocytes définitifs, répartis en quatre systèmes pigmentaires que l'on retrouve jusque chez la grenouille adulte. Anatomiquement, ces quatre manchons pigmentaires sont constitués par un système dermique, un système péricoelomien, un système péri-neural et un système péri-vasculaire. Leur rôle, chez le têtard comme chez la Grenouille, est évidemment en rapport étroit avec la régulation thermique et lumineuse. En effet, le tissu conjonctif du batracien n'élabore pas de tissu adipeux ${ }^{1}$, ce régulateur essentiel de la température chez les homéothermes, mais seulement du pigment.

Les premiers mélanocytes du système dermique commencent à se différencier chez l'embryon pourvu de branchies externes (long. totale: $5 \mathrm{~mm}$.); certaines cellules du derme se détachent du réseau conjonctif et élaborent des granulations pigmentaires. Au début, ces cellules sont massives ne possédant que de courts prolongements; elles acquièrent rapidement tout un système de ramifications, plus ou moins compliquées et chez le têtard venant d'éclore (long. du corps : $3 \mathrm{~mm}$.) leurs ramifications s'insinuent déjà profondément entre les cellules épithéliales, ce qui a pu faire croire à certains auteurs que ces mélanocytes étaient d'origine épidermique.

Les mélanocytes du système péricoelomien sont reconnaissables à un stade un peu plus précoce déjà, chez l'embryon à branchies externes à peine indiquées (long. totale: $4 \mathrm{~mm}$.). La surcharge pigmentaire des cellules mésenchymateuses aplaties de la somatopleure augmente rapidement; celles-ci, fusiformes à l'origine, deviennent volumineuses et présentent de courtes ramifications. Chez le têtard venant d'éclore, ces mélanocytes sont encore séparés les uns des autres, formant un chapelet de grosses cellules noires, bordant le coelome. L'élaboration de pigment se poursuivant, elles arrivent au contact, si bien que chez le gros têtard et la Gre-

1 Exception faite du mésenchyme de la partie antérieure de la bandelette génitale aux dépens de laquelle se différencient les corps adipo-lymphoïdes, dont le métabolisme est en rapport étroit avec celui de la gonade. 
nouille le manchon péricoelomien apparaît sur coupe comme un trait noir, épais et continu.

Les deux derniers systèmes pigmentaires péri-neural et périvasculaire se forment de la même manière, à partir de cellules aplaties d'origine mésenchymateuse qui enveloppent le cœur, les gros vaisseaux, le cerveau, la moelle épinière et les gros nerfs.

Ces deux réseaux sont moins importants que les précédents, ils ne forment pas un revêtement continu, leurs éléments restant toujours espacés les uns des autres. Ces quatre systèmes pigmentaires définitifs sont constitués par des mélanocytes vrais, c'està-dire des cellules qui élaborent du pigment mélanique et qui sont capables de s'étaler ou de se contracter sous l'influence de facteurs physiologiques. L'injection d'extraits post-hypophysaires, en particulier, provoque l'étalement des mélanocytes dermiques (Gineste 1943).

Pendant que s'opère cette différenciation de l'apparail pigmentaire définitif, on assiste à une disparition massive des granulations pigmentaires dans tous les organes embryonnaires. On peut concevoir deux processus distincts pour assurer cette disparition du pigment. Le premier, très général et très facilement décelable, est l'expulsion des granulations pigmentaires qui encombrent les cellules et leur élimination ultérieure de l'organisme par les voies naturelles. Le second consisterait en une transformation sur place des granules noires en une substance chromogène incolore, comparable peut-être à la réaction dopa, telle qu'elle existe dans les cellules basales de l'épiderme humain, par exemple; un processus semblable permettrait d'expliquer la dépigmentation précoce et totale des éléments de la chorde et du squelette.

L'épiderme e mbryonnaire primitif ne comporte qu'une seule couche de cellules bourrées de granulations pigmentaires. Chez l'embryon à branchies externes (long. tot.: $5 \mathrm{~mm}$.), l'épiderme devient bistratifié, toujours extrêmement riche en pigment. Peu avant l'éclosion, on constate, par place dans la couche basale, une raréfaction du pigment pouvant aller jusqu'à sa disparition totale. Par desquamation de l'assise supérieure, la pigmentation disparait dans certaines régions de l'épiderme qui devient tacheté chez le jeune têtard et qui le restera chez la Grenouille. Ultérieurement, la pigmentation du tégument se compliquera par l'apparition de chromocytes dermiques élaborant des 
pigments jaune et rouge et de guanocytes. Nous n'aborderons pas ici cette question.

Le $t u b$ e $n$ e $r$ e $u x$ des embryons très jeunes apparaît complètement noirci par une énorme surcharge pigmentaire qui masque sa structure cellulaire. Au début, tous les neuroblastes sont fortement pigmentés mais, rapidement, dès les derniers stades
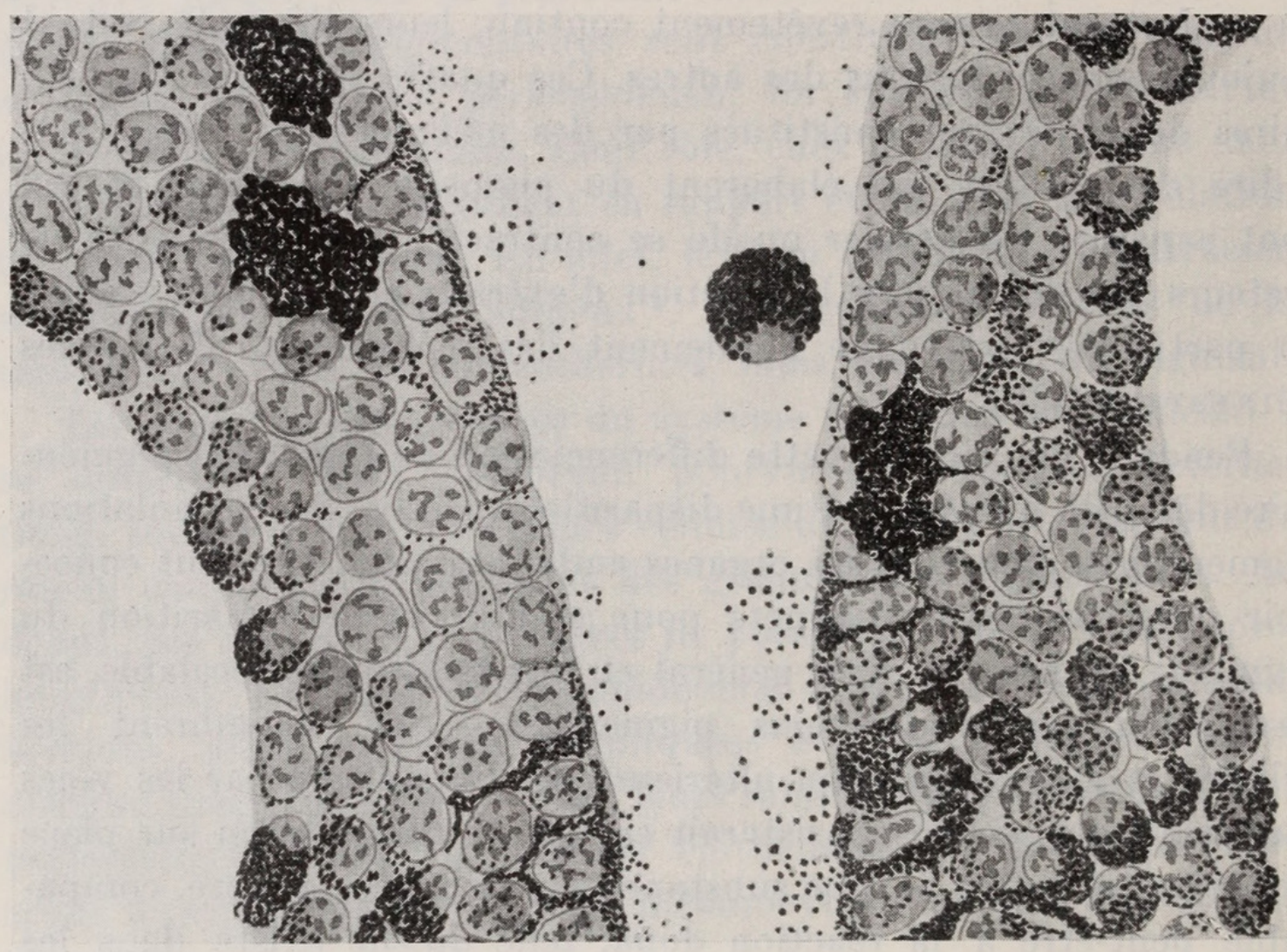

FIG. 1.

Elimination du pigment dans la paroi cérébrale d'un tètard de $R$. esculenta de $5,5 \mathrm{~mm}$. (longueur du corps seulement) (Région de l'aqueduc de Sylvius). Le pigment rejeté par les neuroblastes est soit évacué sous forme de granulations libres, soit capté par de grosses cellules migrantes, qui s'insinuent entre les neuroblastes et finissent par tomber dans la lumière du canal neural (la substance grise a seule été représentée sur la figure). Grossissement: 620 environ.

embryonnaires déjà, on assiste à une élimination massive du pigment. Celle-ci s'effectue selon deux processus différents. Les cellules épendymaires, qui bordent les cavités neurales, rejettent directement dans le liquide céphalo-rachidien le pigment qu'elles renferment, sous forme d'un semis de fines granulations noires. Le pigment des cellules sous-jacentes est déversé dans les interstices 
intercellulaires, formant des travées noires, aboutissant également dans les cavités du tube neural (fig. 1). Enfin, le pigment provenant des couches cellulaires profondes est capté par de très grosses cellules mobiles, munies de prolongements, s'insinuant entre les neuroblastes; elles arrivent peu à peu à la surface épendymaire et sont rejetées dans les cavités neurales qui, à certains moments, peuvent en contenir un très grand nombre. Ce processus d'élimination procède régulièrement d'avant en arrière; chez le têtard, au moment de l'apparition des bourgeons des pattes postérieures (long. du corps: $7 \mathrm{~mm}$.), il est achevé dans la partie céphalique, tandis qu'il se poursuit activement dans la moelle épinière. Il faut noter que la dépigmentation du système nerveux n'est jamais totale; chez la Grenouille, après la métamorphose, les petites cellules nerveuses périphériques de la substance grise contiennent toujours des quantités assez considérables de pigment.

Il nous a été impossible de résoudre deux des problèmes que pose ce processus physiologique. Nous ne pouvons, pour le moment, préciser le mécanisme par lequel le liquide céphalo-rachidien se débarrasse de la masse de granulations pigmentaires libres et des cellules à pigment qu'il renfermait et nous ignorons l'origine de ces grosses cellules. Par leur apparence et leur propriété de fixer le pigment (et les colorants colloïdaux, voir plus loin), elles sont tout à fait comparables aux grands macrophages d'origine mésoblastique qui vont être décrits. Cependant, il nous est impossible de dire si ces cellules à pigment de l'axe nerveux proviennent d'éléments mésenchymateux, qui se seraient infiltrés de bonne heure dans la masse des neuroblastes et qui y exerceraient leur propriété histiocytaire, ou si elles dérivent de cellules nerveuses appartenant à la lignée névroglique, douées de propriétés histiocytaires comme certains éléments du mésenchyme.

Comme nous l'avons signalé précédemment (Du BoIs, 1943) l' intestin est la principale voie d'élimination du pigment. Chez le jeune têtard venant d'éclore (long. du corps: 3-4 mm.) et qui n'a pas encore mangé, la lumière intestinale est remplie, d'un bout à l'autre, par une masse considérable de granulations pigmentaires toujours mélangées, du reste, à une certaine quantité de plaquettes vitellines. Ce processus d'élimination débute dans les derniers stades embryonnaires. Chez l'embryon à branchies externes (long. tot.: $7 \mathrm{~mm}$.), la partie antérieure de l'intestin primitif est 
en voie de se transformer en estomac; les cellules épithéliales, à peu près débarrassées de leurs plaquettes vitellines, sont disposées en une seule couche, aux dépens de laquelle apparaissent les premiers bourgeons glandulaires; ces cellules épithéliales, comme les cellules glandulaires qui en dérivent, contiennent encore chez le

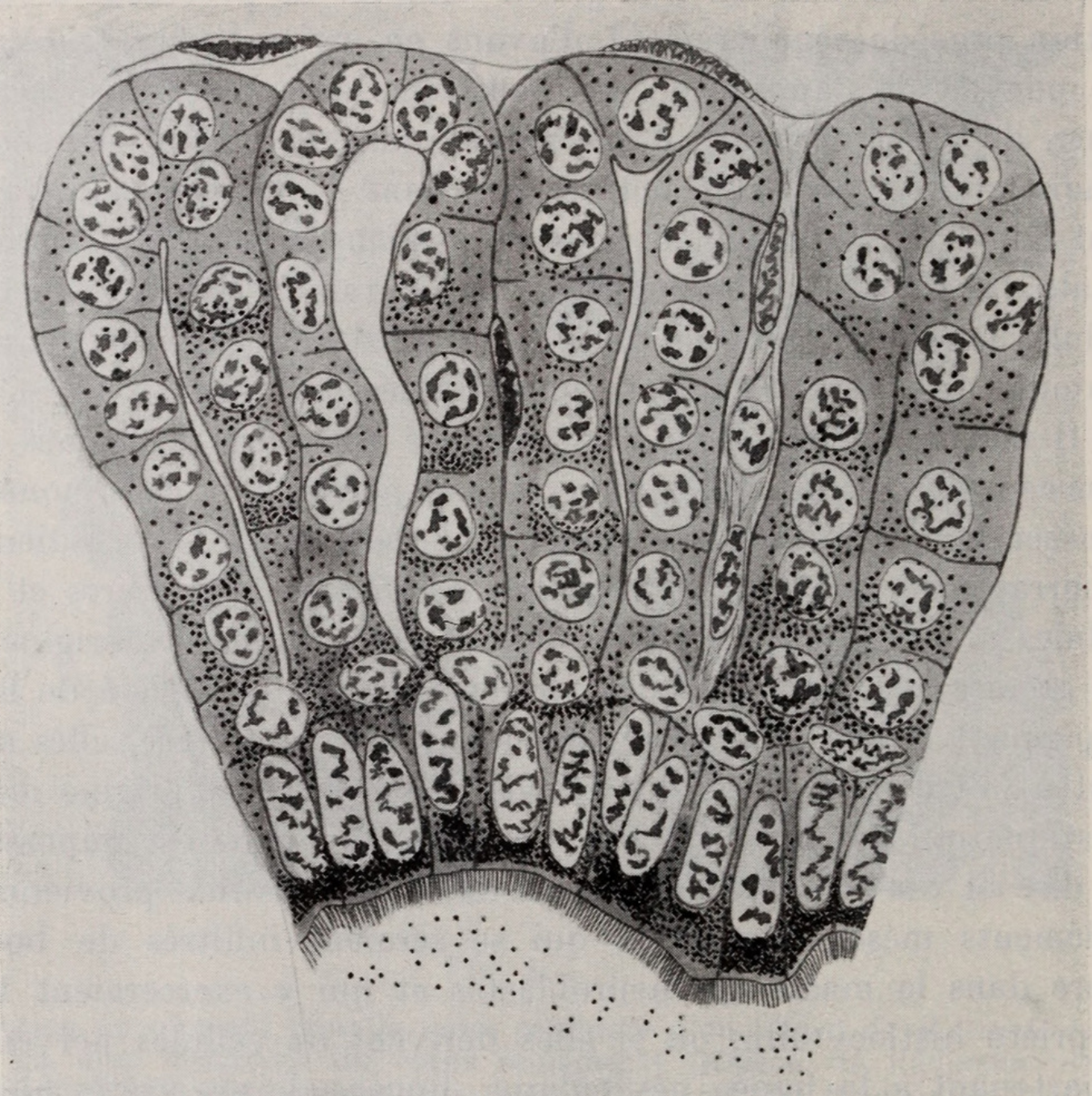

FIG. 2.

Elimination du pigment dans la paroi stomacale d'un têtard de $R$. esculenta de $5 \mathrm{~mm}$. (longueur du corps seulement). Les granulations pigmentaires passent directement d'une cellule glandulaire à l'autre, sans emprunter la voie de la lumière de la glande et sont rejetées dans la cavité gastrique par les cellules épithéliales, ciliées à ce stade. Grossissement: 620 environ.

têtard après l'éclosion une certaine quantité de granulations pigmentaires (fig. 2); celles-ci sont refoulées vers le pôle apical de la cellule épithéliale et expulsées dans la cavité stomacale.

Ce phénomène progresse d'avant en arrière, au fur et à mesure 
de la différenciation du tube digestif. Chez le têtard de 5-6 mm. (long. du corps), l'élimination est presque achevée dans la région stomacale tandis qu'elle se poursuit dans l'intestin; elle est particulièrement active dans l'intestin postérieur. Cette première vague d'élimination massive marche de pair avec la différenciation des régions intestinales et peut être considérée comme l'élimination directe du pigment contenu dans les cellules épithéliales ellesmêmes; en effet, l'élimination cesse au niveau de l'estomac, dès que les cellules qui constituent sa paroi se sont débarrassées de leur surcharge pigmentaire, tandis qu'elle se poursuit dans l'intestin durant toute la vie larvaire.

La paroi de l'intestin spiralé, caractéristique du têtard, est excessivement simple. Elle est constituée, sur toute sa longueur, par une couche de cellules prismatiques élevées, ciliées dans certaines régions chez les très jeunes têtards et à plateau bien dessiné dans les stades plus âgés. Cet épithélium, qui renferme quelques cellules muqueuses, est entouré d'une tunique conjonctive très mince, recouverte par le mésothélium péritonéal. La lame conjonctive renferme fréquemment d'énormes cellules migrantes, à pseudopodes, bourrées de granulations pigmentaires. Arrivées sous l'épithélium, elles se déchargent peu à peu de leur contenu, les granulations pigmentaires passent dans les cellules épithéliales adjacentes et s'accumulent sous le plateau; celui-ci devient peu net (fig. 3), prend une apparence boursouflée, les granulations filtrent à travers cette zone et sont libérées dans la cavité intestinale.

Cette fonction excrétrice de la cellule intestinale n'est que transitoire et disparaît au moment de la métamorphose. Lorsque le long intestin spiralé du têtard herbivore se transforme en intestin court de la Grenouille carnivore, la cellule intestinale devient imperméable aux granulations pigmentaires. La métamorphose de l'intestin débute immédiatement après l'apparition des pattes antérieures, c'est-à-dire chez le têtard de $13 \mathrm{~mm}$. (long. du corps). Une mince lame musculaire se différencie à la périphérie de la tunique propre, puis tout l'intestin se contracte en formant de gros plis. Les cellules épithéliales se multiplient rapidement et desquament en grands lambeaux que l'on retrouve dans la lumière intestinale, si bien que l'on a pu parler d'une "mue intestinale " (Duesberd, 1906; Janes, 1934). Cette métamorphose se fait en 48 heures approximativement, pendant lesquelles l'animal ne 
se nourrit pas. Comme la régression de la queue s'effectue pendant le même laps de temps, une masse assez considérable de pigment doit être rapidement éliminée. Les gros macrophages,

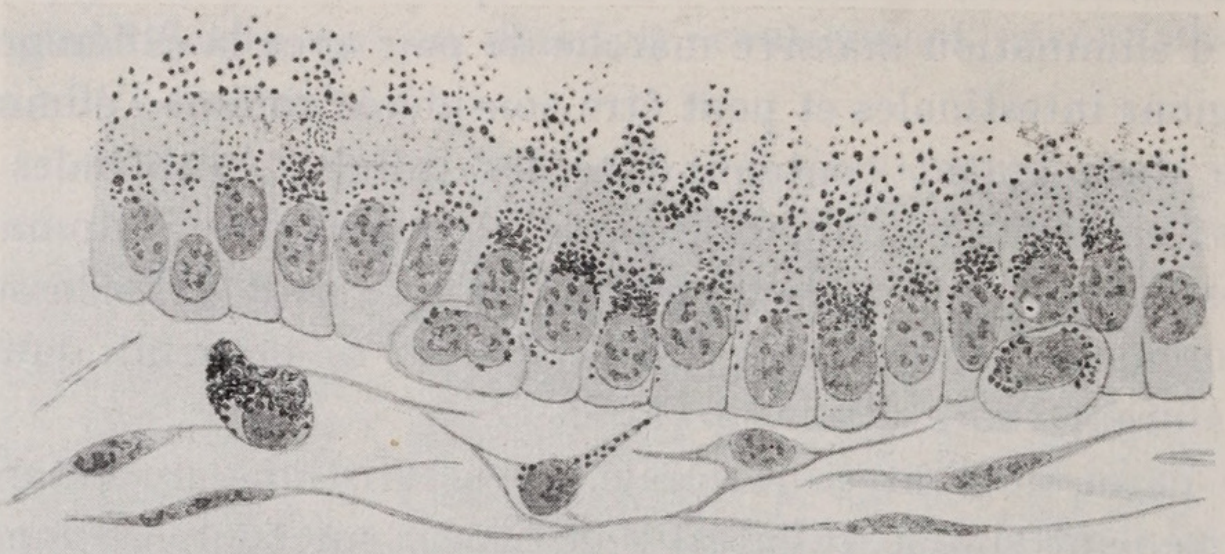

FIG. 3

Elimination du pigment à travers l'épithélium intestinal d'un jeune têtard de $R$. esculenta de $7 \mathrm{~mm}$. (longueur du corps seulement). Les gros macrophages migrants se déchargent de leur pigment dans les cellules épithéliales qui le rejettent dans la cavité intestinale. Durant ce processus, le plateau cellulaire s'efface, remplacé par une zone aux limites imprécises à travers laquelle le pigment filtre sous forme de granulations excessivement fines. Grossissement: 620 environ.

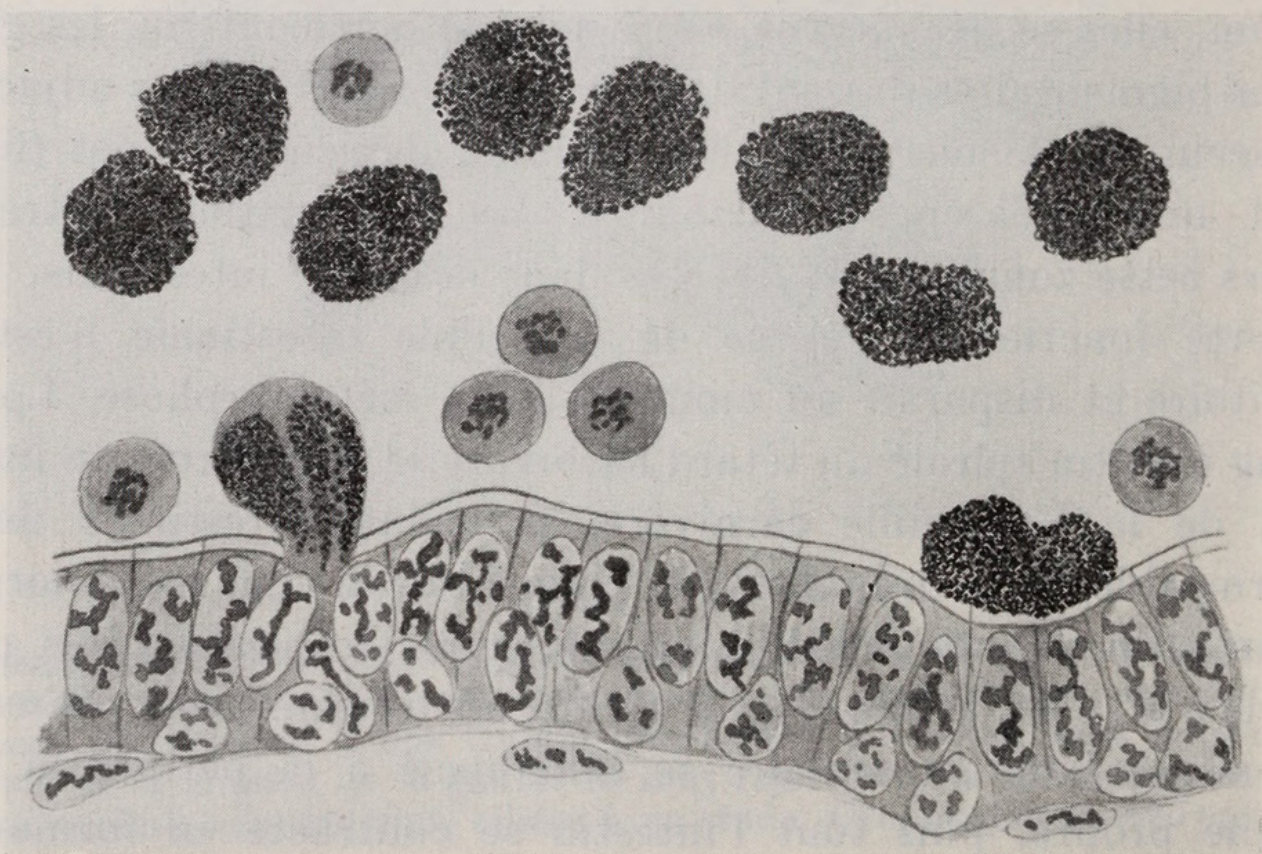

FIG. 4.

Elimination des macrophages chargés de pigment à travers la paroi intestinale pendant la métamorphose (têtard de $R$. temporaria à queue très réduite et à intestin déjà court du type Grenouille). La lumière intestinale contient, à côté des macrophages, une quantité de cellules en dégénérescence provenant de la desquamation de l'épithélium larvaire. Grossissement: 620 environ. 
au lieu de déverser leurs granulations dans les cellules intestinales, s'insinuent entre celles-ci et sont rejetés dans la cavité intestinale, qui se remplit de grosses cellules noires (fig. 4). Cette expulsion de cellules pigmentaires à travers la paroi intestinale serait le mode normal d'élimination du pigment chez la Grenouille adulte (ENRIQuEz, 1904), mais après la métamorphose le processus est extrêmement ralenti.

L'origine de ces macrophages que l'on rencontre, du reste, en petit nombre dans la paroi intestinale de la Grenouille adulte, est discutée. Pour Krause (1923), ces cellules seraient des athrocytes libérés des îlots pigmentaires du foie tandis qu'ENRIQUEZ (1904) les considère comme des macrophages ordinaires, d'origine conjonctive. Chez le têtard, il est vraisemblable que ces cellules amènent dans la paroi intestinale le pigment à éliminer, recueilli un peu partout dans l'organisme. On trouve, en effet de ces cellules pigmentaires migrantes dans toutes les régions du corps, en particulier dans le voisinage immédiat de la musculature en voie de dépigmentation (fig. 5). L'origine conjonctive de certains de ces macrophages paraît indubitable, d'autres doivent certainement provenir des îlots pigmentaires du foie (voir plus loin). Le problème du pigment hépatique et sang u in reste encore à élucider. L'ébauche du foie apparait chez la neurula âgée et les cellules qui la constituent sont, dès l'origine, bourrées de granulations pigmentaires. La structure hépatique n'apparaît 
que chez l'embryon à branchies internes (long. tot.: $7-8 \mathrm{~mm}$.). Les travées hépatiques, formées de cellules richement pigmentées, délimitent un réseau capillaire, généralement très dilaté; ici et là, des cellules beaucoup plus noires doivent être homologuées aux cellules de von KuPfFER, c'est-à-dire à ces cellules de la paroi capillaire douées de propriétés histiocytaires et capables de capter les granulations pigmentaires. Ces cellules drainent tout d'abord le pigment contenu dans les cellules hépatiques embryonnaires, car leur pigment n'est certainement pas évacué dans l'intestin par la voie biliaire; en effet, ni les canaux biliaires, ni la vésicule biliaire, proportionnellement très volumineuse chez le jeune têtard, n'en renferment à aucun moment (cette conclusion sera confirmée dans la partie expérimentale). Chez le têtard de 6-7 mm. (long. du corps) les cellules hépatiques sont presque complètement débarrassées de leur pigment.

Il est possible que les cellules de von KupfFer captent également les granulations pigmentaires libres qui circulent dans le sang (on en trouve surtout au moment de la résorption de la queue) et le pigment des cellules sanguines. Les cellules sanguines primitives, décrites par Maxımow (1927), se présentent chez l'embryon pourvu de branchies externes (long. tot.: 7,5 mm.) comme de grosses cellules rondes, bourrées de pigment et de plaquettes vitellines; chez l'embryon prêt à éclore, ces cellules primitives ont donné naissance à des érythroblastes, ayant déjà acquis la forme ovalaire des érythrocytes définitifs, mais qui renferment encore des grains de pigment et un certain nombre de plaquettes vitellines. Il est probable que ces cellules se déchargent de leur pigment, et peut-être aussi de leur vitellus, au niveau du foie. Chez le jeune têtard (long. du corps: 4-5 mm.), les érythrocytes dépourvus de plaquettes vitellines ne contiennent plus que quelques granulations pigmentaires qui ont complètement disparu chez le têtard de 9-10 mm. (long. du corps).

Lorsque l'élimination du pigment embryonnaire est achevée, les cellules de von Kupffer jouent un rôle dans l'élimination du pigment d'origine hépatique, provenant de la destruction des hématies. Prenant (1909) a, en effet, constaté la présence de restes d'érythrocytes dans les cellules pigmentaires de von KupfFER à faible surcharge granulaire du foie de la Grenouille adulte. Ces cellules, une fois bourrées de pigment, restent probablement en 
place pendant assez longtemps. Elles finissent par se détacher de la paroi endothéliale et par passer dans la circulation, ainsi que l'a constaté Herzog (1924), chez la Grenouille adulte. Ces cellules ne sont donc pas des mélanocytes, au sens exact du terme, car elles n'élaborent pas de pigment mais se bornent à l'emmagasiner. Elles ne réagissent pas à l'hormone post-hypophysaire comme les mélanocytes vrais (Gineste, 1943). Groupées en petits amas, elles forment des îlots pigmentaires du foie du têtard et de la Grenouille adulte. Le nombre de ces îlots pigmentaires est très variable suivant les individus; il change probablement selon les conditions physiologiques.

Il existe une seconde voie d'élimination du pigment: le p r o $\mathrm{n}$ é p h r o s. Cette voie de transit, également utilisée pour l'évacuation des colorants colloïdaux d'origine exogène, a fait l'objet d'études très détaillées de von Moellendorff (1919) et GÉrard et Cordier (1933) et ne sera pas décrite à nouveau ici. Il nous suffira de signaler que les granulations pigmentaires s'accumulent dans les cellules épithéliales des canaux du pronéphros chez l'embryon de 7-8 mm. (long. tot.) déjà, ce qui semble indiquer que le rein primitif est fonctionnel chez la larve, avant l'éclosion (ce fait sera confirmé plus loin). Les différents segments du tube urinaire pronéphridien paraissent tous capables de capter les granulations pigmentaires et en contiennent des quantités considérables, chez la larve prête à éclore et chez les très jeunes têtards, au moment de l'élimination massive du pigment embryonnaire. Cette surcharge pigmentaire diminue progressivement et, au moment où le pronéphros entre en régression (têtards de 10-11 mm., long. du corps), les cellules n'en contiennent plus qu'une quantité réduite. Chez l'embryon prêt à éclore et les jeunes têtards, les glomérules pronéphridiens renferment toujours passablement de pigment et se présentent, sur coupes, comme de petits organes très noirs. Il semble que le pigment y soit retenu ou qu'en tout cas, il ne filtre que très lentement à travers la paroi glomérulaire. 


\section{ATHROCYTOSE ET ÉLIMINATION DES COLLOÏDES D'ORIGINE EXOGENE AUX DIFFÉRENTS STADES EMBRYONNAIRES ET LARVAIRES}

I. Premières manifestations de L'activité histiocytaire.

A. Injections au stade neurula.

Les neurulas de $R$. temporaria, ayant reçu une injection de bleu trypan ou de bleu de Prusse, ont été fixées, soit après 48 heures à un stade où le corps s'est allongé par l'apparition du bourgeon caudal (long. tot.: $4 \mathrm{~mm}$.), soit après 72 heures au moment où les branchies externes commencent à apparaître (long. tot.: 4,5-5 mm.). Dans ces séries d'embryons très jeunes, il y a toujours une assez forte proportion d'individus opérés qui ne présentent pas trace de colorant dans leurs tissus (par exemple dans la série 4, injectée de bleu de Prusse, 10 individus sur 23 ne renfermaient pas trace de bleu; la proportion est à peu près la même dans les autres séries); cela est dû au fait que le colorant a probablement été expulsé par la plaie.

Dans les cas les plus démonstratifs, fixés après 72 heures, l'examen histologique montre que le colorant forme des traînées et des flaques bleues dans le coelome, autour de l'énorme intestin. On rencontre fréquemment; dans ces plages colorées, de petits groupes de cellules sanguines primordiales, arrondies, riches en pigment et en plaquettes vitellines. A ce stade très précoce, aucune cellule embryonnaire n'est encore capable de capter le colorant, qu'il s'agisse du bleu trypan ou du bleu de Prusse; la fonction histiocytaire n'a pas encore apparu. A la suite des lésions fréquentes de la paroi intestinale, dues à la piqûre, on constate la présence de dépôts de colorant dans cette paroi et une imprégnation bleue de quelques cellules intestinales riches en vitellus, plus ou moins déchirées par l'intervention. I] s'agit là d'une imbibition de cellules mortes qui n'a évidemment rien à voir avec le phénomène d'athrocythose. 
B. Injections d'embryons ayant un bourgeon caudal bien développé. (Long. totale: $4,5 \mathrm{~mm}$.)

Au moment de la fixation, soit 24 heures après l'intervention, les embryons sont devenus très mobiles et possèdent des branchies externes bien développées (long. tot.: 5,5-6 mm.). On retrouve, comme au stade précédent, le colorant sous forme de traînées dans le coelome; celles-ci contiennent toujours un certain nombre de cellules sphériques, riches en granulations pigmentaires mais qui paraissent avoir perdu déjà une partie de leur surcharge vitelline. Quelques-unes de ces cellules ont capté un peu de colorant (bleu de Prusse) et renferment quelques granulations bleues à côté des granulations pigmentaires. Ces cellules, très vraisemblablement des cellules sanguines primitives, sont donc les premiers éléments qui manifestent des propriétés histiocytaires.

D'autre part, à ce stade, la différenciation du pronéphros est déjà assez avancée et, dans un cas, il présentait même un début d'activité fonctionnelle; les cellules épithéliales des tubes pronéphridiens renfermaient, à côté d'une grosse surcharge pigmentaire, quelques granulations de bleu de Prusse.

\section{Injections d'embryons mobiles, ayant des branchies externes} bien développées.

(Long. totale: 5-6 mm.)

Les embryons ont été fixés, soit 24 heures après l'injection (long. tot.: $6-7 \mathrm{~mm}$.), soit 48 heures après (larves prêtes à éclore, long. tot.: $8 \mathrm{~mm}$.). On constate que les cellules sanguines athrocytaires ayant accumulé des granulations bleues sont devenues plus nombreuses; on en trouve un peu partout. En outre, on assiste à une élimination massive du colorant injecté (bleu de Prusse ou bleu trypan suivant les séries) et de pigment par le pronéphros; la lumière des tubes urinaires renferme du bleu et des granulations pigmentaires et les cellules épithéliales sont bourrées de granulations bleues et noirès. Si l'injection a été faite très latéralement, le bleu est exclusivement capté par le rein situé du côté de la piqûre.

Le foie qui, chez le têtard, jouera un rôle important dans le phénomène d'athrocytose, n'est encore qu'une ébauche massive dans laquelle les cellules endothéliales ne manifestent pas encore

Rev. Suisse de Zool., T. 53, 1946. 
d'activité histiocytaire. Si parfois un peu de colorant pénètre dans les vaisseaux hépathiques, il reste extracellulaire.

E n r és u m é les injections de colorant pratiquées sur les embryons montrent que les premiers signes d'une activité athrocytaire n'apparaissent que chez l'embryon de 5,5 mm., caractérisé par sa forme allongée, ses branchies externes et sa grande mobilité. A ce stade, cette fonction est uniquement assurée par quelques cellules sanguines arrondies, renfermant du pigment en assez grande quantité et des plaquettes vitellines. Il semble que ces cellules ne puissent devenir des athrocytes que lorsque leur surcharge vitelline a quelque peu diminué. Elles existent en effet déjà au stade précédent, se trouvant même souvent en contact direct avec le colorant, sans pouvoir le fixer.

Plus tard, le nombre de ces cellules athrocytaires, plus ou moins chargées de bleu, va en augmentant et l'on en rencontre un peu partout dans l'organisme. Elles captent avec la même facilité, semble-t-il, les grosses particules du bleu de Prusse et les petites du bleu trypan.

En outre, ces expériences montrent que le pronéphros commence déjà à fonctionner chez l'embryon de 5 à $6 \mathrm{~mm}$.; la fixation des premières granulations bleues par les cellules épithéliales des tubes urinaires coïncide avec l'accumulation des granulations pigmentaires; ce résultat vient ainsi confirmer la constatation faite plus haut à propos du pigment. Dans son travail sur l'élimination du bleu trypan par le pronéphros du têtard, von Moellendorff (1919) admet que cette fonction doit débuter peu après l'éclosion, mais les expériences qui viennent d'être relatées prouvent que l'activité physiologique du pronéphros commence à se manifester à un stade beaucoup plus précoce.

II. Athrocytose et Élimination des Collö̈des exogènes CHEZ LE TÊTARD, DE L'ÉCLOSION A LA MÉTAMORPHOSE.

Pour faciliter l'exposé des résultats expérimentaux, nous traiterons séparément la question des athrocytes et celle des voies d'élimination.

A. Les cellules athrocytaires.

Les cellules sanguines, à fonction histiocytaire, signalées dans les stades embryonnaires, passent au second plan chez le têtard, 
tandis que les cellules de von KupfFer du foie jouent un rôle de plus en plus prépondérant dans le phénomène de la colloïdopexie. Elles captent (fig $6 a$ ) indistinctement toutes les particules colloïdales circulant dans l'organisme, aussi bien la granulation minuscule du bleu trypan que l'énorme particule d'encre de Chine.

Dans nos diverses séries d'expériences, tout le colloïde en circulation a déjà été retenu par le foie 24 heures après l'injection. L'activité histiocytaire des cellules de von KupfFer doit débuter très rapidement après l'injection, dès que le colloïde est entré dans la circulation; il faudrait fixer les animaux peu après l'injection pour savoir si la cellule endothéliale capte avec la même rapidité les particules de grosseurs différentes. Toutes les cellules endothéliales du foie sont capables d'athrocytose et après injection de doses massives de l'un des colloïdes, le foie paraît absolument farci de cellules de von KupfFer, plus ou moins bourrées de granulations. Cette surcharge en colorant se traduit, à l'échelle macroscopique au moment de la dissection, par l'aspect du foie tacheté de bleu ou de noir. Parmi les cellules des îlots pigmentaires, celles qui au moment de l'injection ne contenaient pas beaucoup de pigment, captent une certaine quantité de colloïde et apparaissent, sur coupe, bourrées d'un mélange de granulations noires et bleues (lorsqu'il s'agit d'injection d'encre de Chine, une dépigmentation peu poussée conserve une teinte brun clair aux granulations pigmentaires, qui se reconnaissent ainsi facilement des particules de l'encre de Chine). Ce résultat vient confirmer le fait déjà signalé dans la première partie, à savoir que les cellules des îlots pigmentaires du foie normal ne sont pas des éléments particuliers, uniquement en relation avec le métabolisme du pigment, mais qu'elles sont des cellules endothéliales douées d'un grand pouvoir athrocytaire, qui fixent indifféremment le pigment ou les colloïdes. Les cellules de von KupfFer chargées de colloïde doivent probablement se libérer à la longue de la paroi capillaire et entrer dans la circulation.

Nous n'avons jamais constaté de signes de dégénérescence du parenchyme hépathique à la suite de l'oblitération des capillaires obstrués par les cellules de von KupfFer hypertrophiées; la durée de l'expérience a vraisemblablement été trop courte. Les cellules hépatiques elles-mêmes ne renferment jamais de granulations colorées, elles semblent absolument imperméables aux particules 
colloïdales, même à celles du bleu trypan. Par conséquent les colloïdes, pas plus que le pigment, ne peuvent être éliminés par la voie biliaire, fait confirmé encore par l'absence totale de colorant dans la vésicule biliaire. Nos observations concordent avec celles de von Moellendorff (1925) sur la Grenouille adulte: la cellule hépatique de Batracien est incapable de capter les particules colloïdales, alors que la cellule hépatique des Mammifères peut parfois contenir du bleu trypan en petite quantité. Le foie n'est donc qu'une voie de garage et non une voie d'élimination naturelle des colloïdes étrangers à l'organisme.

A côté des cellules de von KupfFER, il existe encore plusieurs autres types d'athrocytes. Tous les histiocytes conjonctifs sont capables de fixer les particules colloïdales et, dans le voisinage du point d'injection, on rencontre fréquemment de belles cellules étoilées, plus ou moins chargées de granulations colorées (fig. 6b). Les histiocytes fusiformes de la tunique propre du péritoine et du péricarde sont ceux qui, dans nos expériences, contiennent le plus fréquemment une grosse surcharge granulaire. Cela est probablement dû à la localisation de l'injection plutôt qu'à un pouvoir colloïdopexique particulièrement développé de ces cellules. Les histiocytes fixent indifféremment le bleu trypan, le saccharate de fer et le bleu de Prusse; nous n'avons pas trouvé de figure absolument certaine d'histiocytes chargés d'encre de Chine. L'encre de Chine est certainement plus difficile à fixer par l'organisme que les colloïdes plus dispersés; 48 heures après l'injection, on la retrouve encore, en grande partie, sous forme de vastes nappes entre les organes viscéraux et contre la paroi coelomique; il est difficile de savoir, dans ce cas, si les granulations noires sont englobées dans les cellules histiocytaires ou simplement collées à leur surface.

Dans le sang circulant, au milieu de la foule des érythrocytes on trouve parfois, dans le cœur en particulier, de petites cellules rondes bourrées de granulations bleues (nous n'en avons jamais trouvé contenant de l'encre). Ces cellules sont très vraisemblablement des descendants directs des premiers athrocytes sanguins décrits, plus haut, chez les embryons. L'hémocytoblaste, cellule ronde circulant dans le sang de l'embryon, riche en pigment et en plaquettes vitellines, donnerait donc, d'une part, les érythrocytes ovales, dépourvus de toute propriété athrocytaire et, d'autre part, des cellules rondes, décrites par Maxımow (1927), à cyto- 
plasme légèrement granuleux et à noyau globuleux, rares dans le sang de la Grenouille adulte; ces éléments leucocytaires seraient capables d'athrocytose (fig. $6 d$ ).

Les grosses cellules migrantes, qui jouent un rôle si important dans le transit du pigment, sont aussi des agents athrocytaires

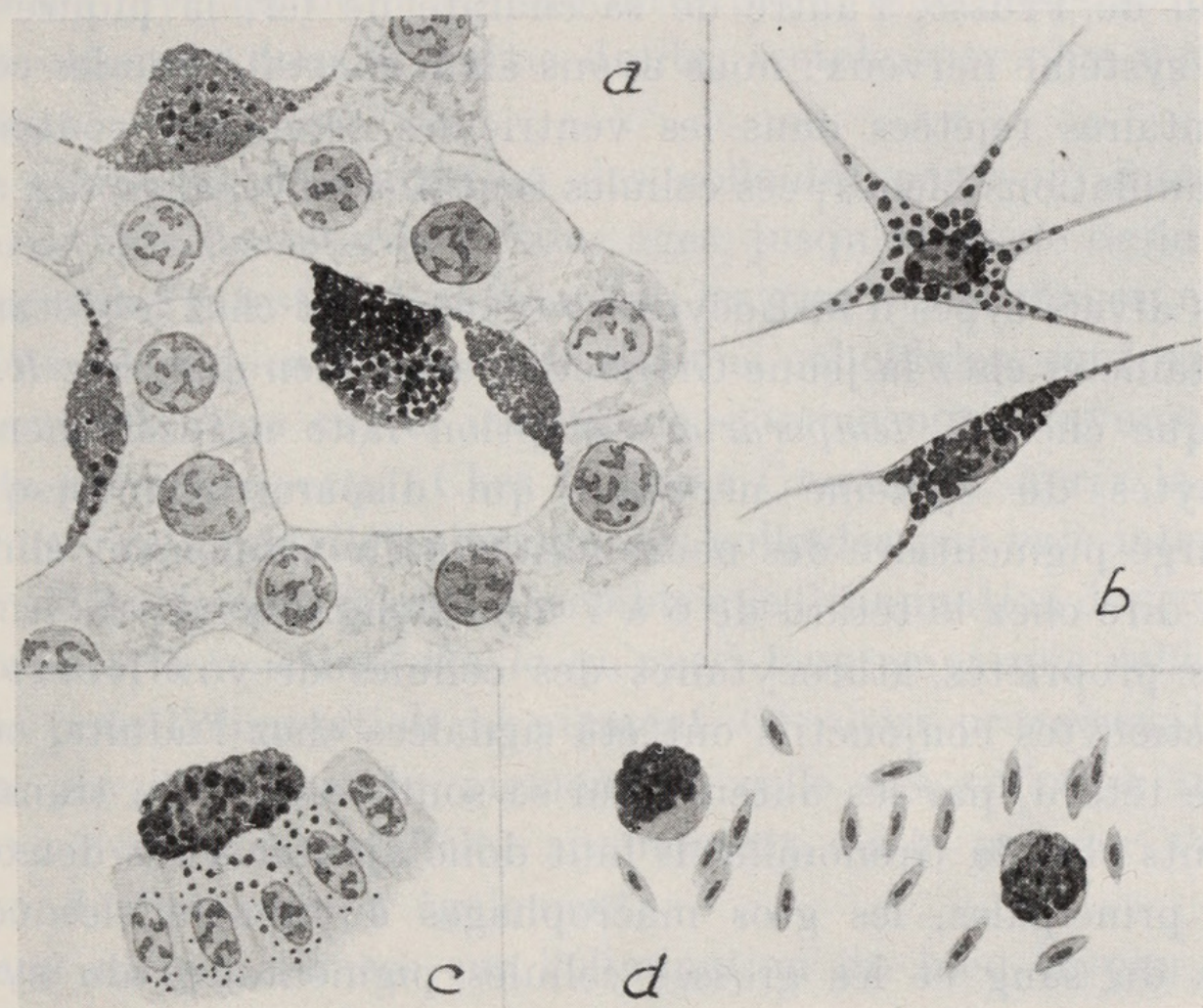

Fig. 6 .

Les principaux types d'athrocytes du têtard.

Les granulations pigmentaires sont figurées en noir, les granulations colorées en gris.

a) Cellules de von Kupffer, dans le foie d'un têtard de $R$. esculenta de $11 \mathrm{~mm}$. (long. du corps) après injection massive de bleu trypan. Toutes les cellules endothéliales sont plus ou moins bourrées de granulations bleues. La cellule pigmentaire, qui fait saillie dans le capillaire, a également capté des particules de colorant. Grossissement: 620 env.

b) Gros histiocytes du tissu conjonctif péribranchial plus ou moins bourrés de granulations colorées. Têtard de $R$. esculenta de $10 \mathrm{~mm}$. (long. du corps), injecté au saccharate de fer. Grosseur: 620 environ.

c) Grosse cellule pigmentaire migrante renfermant à la fois du pigment et des granulations colorées, prête à faire passer sa surcharge granulaire dans les cellules épithéliales de l'intestin. Têtard de $R$. esculenta de $9 \mathrm{~mm}$. (longueur du corps), injecté au saccharate de fer. Grossissement: 620 environ.

d) Cellules sanguines ayant capté du bleu de Prusse. Têtard de R. temporaria de $7 \mathrm{~mm}$. (longueur du corps), injecté au bleu de Prusse. Grossissement: 620 environ. 
très actifs. Après injection de colorants colloïdaux, elles renferment à côté du pigment des granulations colorées en quantité plus ou moins importante (fig. $6 c$ ); on en trouve un peu partout dans l'organisme, en particulier dans la nappe conjonctive de la tunique propre intestinale et au voisinage des canaux pronéphridiens.

Enfin chez deux têtards de 4,5 mm. (long. du corps), l'un injecté de bleu de Prusse, l'autre de saccharate de fer, la piqûre avait lésé le système nerveux; nous avons alors constaté que les cellules pigmentaires rejetées dans les ventricules cérébraux contenaient des granulations bleues; ces cellules sont donc elles aussi des athrocytes.

Ces divers types d'histiocytes se rencontrent chez les têtards de toute taille et chez la jeune Grenouille, aussi bien que chez R. esculenta que chez $R$. temporaria, exception faite naturellement des athrocytes du système nerveux, qui disparaissent lorsque la surcharge pigmentaire des neuroblastes a été totalement éliminée, c'est-à-dire chez le têtard de 6 à $7 \mathrm{~mm}$. (long. du corps).

Les propriétés athrocytaires des cellules de von KupfFer et des histiocytes conjonctifs ont été signalées chez l'adulte, comme chez le têtard, par les auteurs qui se sont occupés du transit des colorants chez la Grenouille. Il faut donc ajouter à ces deux catégories principales, les gros macrophages à pigment, les cellules rondes du sang et les grosses cellules pigmentaires du système nerveux.

\section{B. Elimination des colloüdes.}

Chez le têtard, les colloïdes sont éliminés par les mêmes voies que le pigment, à savoir par l'intestin et le pronéphros.

L'élimination à travers la paroi intestinale se fait selon le mécanisme précédemment décrit pour le pigment: les gros macrophages chargés de granulations colloïdales, généralement mélangées à des granulations pigmentaires, arrivés à la base de cellules épithéliales, y déchargent leurs inclusions; celles-ci passent à travers la cellule épithéliale et sont évacuées dans la cavité intestinale. Le passage des colloïdes à particules petites et moyennes s'effectue rapidement et, après 24 heures, la lumière intestinale renferme toujours une grande quantité de colorant. L'encre de Chine passe plus difficilement; 48 heures après l'injection, l'intestin en contient relativement peu, tandis qu'on retrouve encore de l'encre de Chine 
libre, en flaques, autour de l'intestin et même dans la tunique conjonctive de ce dernier. 48 heures après l'injection d'un mélange à parties égales d'encre de Chine et de bleu de Prusse une assez grande quantité de bleu et fort peu d'encre ont passé dans la lumière intestinale; le bleu traverse donc plus rapidement que l'encre la barrière entérique. Cette élimination des colloïdes peut se faire à tous les niveaux de la spirale intestinale, mais nous ne l'avons jamais observée dans la partie droite, rectale, pas plus que dans l'estomac.

Ce processus d'élimination des colloïdes par voie intestinale s'observe chez les têtards de tous âges, jusqu'au stade de la métamorphose de l'intestin. A ce moment, les gros macrophages, chargés à la fois de pigment et de granulations colloïdales, sont expulsés i n to to dans la cavité intestinale, comme nous l'avons décrit dans la première partie. Chez la jeune Grenouille, après la métamorphose, ce mode d'élimination des colloïdes par voie intestinale est considérablement ralenti. Il semble que l'élimination des colloïdes n'emprunte la voie intestinale qu'aussi longtemps que celle-ci est utilisée pour l'élimination du pigment. Ces deux processus, l'élimination physiologique du pigment et celle des colloïdes injectés ont une évolution parallèle; au moment où le premier s'arrête, le second s'interrompt également.

Dans ses expériences sur l'élimination du bleu trypan par le pronéphros du têtard, von Moellendorff (1919) n'a pas utilisé la technique des injections, mais il a simplement placé des têtards pendant quelques jours dans une solution de bleu trypan à $5 \%$. Après ce traitement, le pronéphros et les cellules de von KupfFER contenaient des quantités appréciables de granulations bleues. Le bleu trypan est donc absorbé par le système digestif. En maintenant les larves de Salamandres pendant 14 jours dans une solution de bleu trypan à $5 \%$, Sснміdт (1931) arrive aux mêmes résultats; Huppert (1926), enfin, étudiant le problème de la résorption du bleu trypan dans le tractus digestif de la Grenouille adulte, constate qu'il est résorbé par l'épithélium et les glandes stomacales surtout et en moindre quantité dans la partie proximale de l'intestin.

Nous avons répété ces expériences avec le bleu trypan et le bleu de Prusse en modifiant quelque peu la technique, car le bleu de Prusse dilué flocule rapidement et se dépose sur le fond du récipient. Nous avons nourri des têtards venant d'éclore ou au 
stade précédant immédiatement la métamorphose, avec une pâtée de semoule additionnée de l'un ou de l'autre des colorants. Les animaux ont été sacrifiés après avoir été nourris de la sorte pendant 4 à 5 jours. Chez les jeunes têtards comme chez les individus plus âgés nourris au bleu trypan, l'épithélium des tubes du pronéphros et les cellules de von KupfFER contenaient des granulations bleues, en petite quantité; cette constatation confirme ainsi les résultats des auteurs cités plus haut. La quantité de bleu trypan ingérée par ce moyen était évidemment très faible; le système digestif ne contenait que peu de bleu et il nous a été impossible de déceler à quel niveau s'opérait l'absorption des particules de colorant. Par contre, les têtards nourris au bleu de Prusse n'ont jamais présenté la moindre trace de colorant, ni dans le foie, ni dans le rein primitif; les particules de bleu de Prusse ne sont pas absorbées dans l'épithélium digestif.

Ces expériences montrent que la cellule épithéliale de l'intestin jouit chez la Grenouille, pendant la vie larvaire et dans certaines conditions, d'une bipolarité fonctionnelle; elle est capable d'absorber et d'éliminer divers colloïdes. - Son pouvoir absorbant est limité aux colloïdes finement dispersés (bleu trypan) tandis que sa capacité d'élimination se manifeste aussi vis-à-vis des particules plus grosses.

Le pronéphros constitue la seconde voie d'élimination des colloïdes. Ce mécanisme a été minutieusement étudié par l'élimination du bleu trypan chez le têtard de Grenouille par von MoelLENDORFF (1919) d'une part et pour toute une série de colloïdes chez le têtard de Grenouille et de Crapaud par Gérard et Cordier (1933). Nos résultats sont entièrement superposables à ceux de ces auteurs: le bleu trypan, le saccharate de fer et le bleu de Prusse sont captés par les cellules du segment principal du tube pronéphridien. Ces cellules paraissent avoir quelques difficultés à fixer les grosses particules d'encre de Chine; lorsque la dose d'encre injectée est faible, elle n'en contiennent jamais; si l'injection a été massive, les cellules du segment principal de pronéphros renferment un petit nombre de particules d'encre.

Gérard et Cordier (1932b) sur le Crapaud adulte n'ont jamais constaté de dépôt de granulations d'encre de Chine (de fabrication allemande) dans le segment principal. On peut penser que l'encre de Chine de Grübler, avec laquelle nos expériences ont été faites 
possède des particules plus petites que les encres Perle, Pélikan 551, utilisées par ces auteurs. Comme nous l'avons signalé à propos du pigment, le pronéphros présente déjà des signes incontestables d'activité pendant la vie embryonnaire (embryon à branchies externes, long. tot.: $6 \mathrm{~mm}$.). Dès ce moment, les cellules des tubes urinaires accumulent du pigment et peuvent fixer des particules colloïdales (bleu trypan ou bleu de Prusse). La disposition particulière du pronéphros de la Grenouille permet aux colloïdes injectés de passer directement du coelome dans l'entonnoir cilié du néphrostome, sans emprunter la voie sanguine aboutissant aux glomérules. Ceux-ci, au nombre de trois, sont en effet indépendants des tubes néphridiens et font hernie dans la cavité coelomienne, à quelque distance du néphrostome correspondant. La paroi du glomérule ne laisse passer que les très petites particules du bleu trypan; elle retient déjà celles du saccharate de fer. Certains têtards, injectés avec des doses massives de saccharate de fer, par exemple, présentent une hypertrophie très accusée des glomérules, comme si tout le colorant amené par voie sanguine, y restait bloqué. Le même fait a été constaté avec le bleu de Prusse et le pigment.

Le pronéphros commence à dégénérer chez le têtard de $8 \mathrm{~mm}$. (long. du corps); les cellules du segment principal s'aplatissent, leurs limites s'effacent et leur surcharge en granulations pigmentaires et colloïdales diminue peu à peu. Cette voie d'élimination a complètement cessé de fonctionner au moment de la métamorphose.

Elle est partiellement remplacée par l'élimination à travers le mésonéphros; l'ébauche du rein définitif (GAUPP, 1904) apparaît chez l'embryon prêt à éclore, mais il ne commence à fonctionner que chez le têtard de $8 \mathrm{~mm}$. (long. du corps). La partie initiale des tubes urinaires du mésonéphros ne s'ouvre pas dans le coelome par un néphrostome. On trouve bien, à la face ventrale du rein de la Grenouille adulte et du têtard, quelques néphrostomes, mais ceux-ci n'entrent à aucun moment en rapport avec le tube urinaire; ils se mettent en communication avec les capillaires veineux du réseau péritubulaire (von Moellendorff, 1919; Gérard et Cordier 1933). Ainsi la circulation glomérulaire et le filtrage à travers la paroi viscérale de la capsule de Bowmann forment la seule voie d'accès aux tubes urinaires. Le mésonéphros ne peut éliminer que les colloïdes à particules assez petites pour passer 
à travers le filtre glomérulaire. Ici encore nos résultats concordent tout à fait avec ceux des auteurs déjà cités: le bleu trypan et le saccharate de fer passent à travers le glomérule et sont ensuite captés par le segment principal, tandis que le bleu de Prusse est retenu dans le glomérule. Les tubes du mésonéphros ne contiennent jamais de pigment mais certains glomérules peuvent en renfermer des traces; le pigment semble également incapable de passer le filtre glomérulaire. Le mésonéphros est donc un émonctoire sélectif, capable d'éliminer seulement les colloïdes à petites particules.

On peut se demander par quel mécanisme la Grenouille adulte se débarrasse des colloïdes à grosses particules, puisque le rein définitif en est incapable et que l'élimination intestinale paraît s'être arrêtée à la métamorphose.

\section{CONCLUSIONS}

Les résultats expérimentaux qui viennent d'être exposés permettent d'arriver aux conclusions suivantes:

A. Les colloïdes d'origine exogène sont captés par les mêmes cellules et éliminés par les mêmes voies que le pigment d'origine endogène. L'athrocytose ne semble donc pas être une réponse de l'organisme à un état pathologique ou expérimentalement réalisé; elle est une fonction physiologique normale, en activité constante, qui assure l'élimination de certains déchets du métabolisme. L'élimination massive du pigment dès la fin de la vie embryonnaire et durant toute la vie larvaire, jusqu'au moment de la métamorphose, démontre que ce pigment doit être considéré comme un déchet organique. L'œuf de Grenouille renferme une certaine quantité de pigment qui, au cours de la gastrulation se trouve essentiellement réparti dans l'ectoblaste et le mésoblaste; l'endoblaste en est à peu près dépourvu. Cette quantité initiale de pigment, répartie dans les ébauches de divers organes, est relativement faible. On doit admettre qu'au cours de leur développement les cellules embryonnaires élaborent du pigment, étant donné les quantités énormes de granulations pigmentaires rejetées par l'organisme. Cette élaboration du pigment paraît être en rapport étroit avec le métabolisme des plaquettes vitellines. La cellule intestinale, par exemple, bourrée de plaquettes vitellines et quasi dépourvue de pigment au stade neurula, devient de plus en plus 
noire, au fur et à mesure de la diminution des plaquettes vitellines. Il est impossible d'évaluer d'une manière précise la quantité de pigment contenu dans un tissu particulier, mais étant donné les masses de pigment éliminées pendant le vie larvaire, on en peut déduire que l'embryon doit en élaborer des quantités considérables, qui jouent très vraisemblablement un rôle dans la régulation thermique, avant d'être éliminées.

B. Nos expériences mettent en évidence plusieurs degrés dans le pouvoir sélectif des cellules en fonction de la dimension des particules colloïdales. Les cellules qui jouent le principal rôle dans la fonction athrocytaire, cellules de von KupfFER et grosses cellules migrantes, ne manifestent pas de propriété sélective vis-à-vis des colloïdes et du pigment; elles sont capables de capter indifféremment tous les colloïdes quelle que soit la grosseur de leur particule. Les cellules de l'épithélium intestinal et du segment principal du pronéphros manifestent déjà un certain pouvoir de sélection, en ce sens que bien qu'elles soient capables également de capter les particules de bleu trypan, de saccharate de fer et de bleu de Prusse, elles paraissent n'absorber celles d'encre de Chine qu'avec une certaine difficulté. Ce pouvoir de sélection est encore plus accusé pour les histiocytes et les cellules sanguines qui paraissent incapables de fixer l'encre de Chine. Mais c'est dans les processus de filtrage à travers les glomérules rénaux que la grosseur des particules joue un rôle essentiel: la paroi du glomérule pronéphridien ne laisse passer que le bleu trypan, tandis qu'elle retient le saccharate de fer, le bleu de Prusse et l'encre. Ce fait est démontré par l'hypertrophie souvent énorme des glomérules gonflés par la rétention du colloïde. En outre, les glomérules contiennent toujours une quantité assez considérable de pigment qui semble y être également retenu et qui reste sur place lors de l'involution des glomérules; une tache noire signale encore, chez la jeune Grenouille, l'emplacement qu'occupait chaque glomérule. Par contre, la paroi viscérale du glomérule du mésonéphros est perméable à la fois au bleu trypan et au saccharate de fer, que l'on retrouve plus loin captés dans les cellules du segment principal du tube urinaire. Quelques glomérules peuvent contenir une certaine quantité de bleu de Prusse ou de pigment mais, ni l'un ni l'autre ne passe le filtre glomérulaire; nous n'avons jamais trouvé d'encre de Chine dans un glomérule 
mésophridien. Ces constatations nous permettent de tirer les conclusions suivantes en ce qui concerne la grosseur des particules pigmentaires: elles doivent très vraisemblablement avoir un diamètre supérieur à celles du saccharate de fer puisqu'elles ne filtrent pas à travers le glomérule du mésonéphros. D'autre part ce ne sont certainement pas de très grosses particules, de l'ordre de celles de l'encre de Chine, étant donné la facilité avec laquelle elles traversent en masse l'épithélium intestinal et sont captées par les différents types d'athrocytes, y compris les histiocytes. Dans nos expériences, le pigment et le bleu de Prusse se sont comportés d'une manière tout à fait comparable, mais ce fait ne permet pas de déduire exactement le diamètre des particules de pigment. Tout ce que nous pouvons dire c'est que leur grosseur est certainement supérieure à $24,6 \AA$, dimension des particules de saccharate de fer et inférieure à celle des particules d'encre de Chine; ces dernières, étant à la limite de la visibilité, doivent être voisines de $1000 \AA$.

C. L'élimination du pigment et des colorants colloïdaux à travers la paroi intestinale est une démonstration de l'hypothèse généralement admise de la bipolarité de la cellule intestinale. Chez le têtard, celle-ci fonctionne dans le sens apical-basal au cours $\mathrm{du}$ processus d'absorption et dans le sens basal-apical lors de l'élimination physiologique du pigment ou expérimentale des colloïdes. La disparition des limites du plateau pendant l'élimination du pigment semble indiquer que le fonctionnement de la cellule en direction basale-apicale s'accompagne de modifications de la constitution cellulaire, qui paraissent incompatibles avec la fonction d'absorption. Il est vraisemblable que la cellule intestinale ne peut fonctionner simultanément dans les deux directions; comme nous l'avons signalé, l'élimination du pigment se fait par zones, alternant probablement avec des zones d'absorption. Après la métamorphose, la cellule intestinale de la Grenouille semble perdre sa polarité basale-apicale, du moins en ce qui concerne le passage de granulations pigmentaires et colloïdales. 


\section{RÉSUMÉ}

L'injection de divers colorants colloïdaux dans la cavité coelomienne d'embryons et de têtards de Grenouille a donné les résultats suivants:

1. Les premières manifestations de l'activité athrocytaire ont été constatées chez les embryons à branchies externes, de $6 \mathrm{~mm}$. de longueur totale. Les cellules sanguines primordiales sont les premiers athrocytes fonctionnels.

L'activité du pronéphros débute au même stade; les cellules de l'épithélium du tube urinaire captant dès ce moment les particules de colorant.

2. Le pigment embryonnaire et les colloïdes injectés sont fixés par les mêmes cellules et éliminés par les mêmes voies.

3. Les cellules de von Kupffer du foie, les grosses cellules pigmentaires migrantes -- qui sont peut-être des cellules de von KupfFer libérées --.. et certains éléments sanguins constituent Jes trois types principaux d'athrocytes du têtard. Les histiocytes conjonctifs et certaines cellules pigmentaires du cerveau possèdent également des propriétés athrocytaires.

4. Le pigment et les colorants colloïdaux sont éliminés par les voies intestinales et rénales.

5. Chez l'embryon prêt à éclore, comme chez le têtard de tout âge, la cellule intestinale capte les granulations pigmentaires et colloïdales, amenées à sa base par les athrocytes migrants et les expulse par son pôle apical. Après la métamorphose de l'intestin, les cellules intestinales perdent cette propriété et les athrocytes migrants bourrés de granulations pigmentaires et colloïdales s'insinuent entre les cellules épithéliales et tombent dans la lumière de l'intestin.

6. Le pronéphros sert également de voie d'élimination au pigment et aux colloïdes pendant la première partie de la vie larvaire. Le mésonéphros n'élimine que les colloïdes à très petites particules, ceux à particules plus grosses étant retenus dans le glomérule. 


\section{BIBLIOGRAPHIE}

$1942 a$ Du Bors, A. M.: Contribution à l'étude de la colloïdopexie chez l'Escargot. C. R. Soc. Phys. et Hist. nat. Genève, vol. 59, p. 41-45.

$1942 b-$ Colloüdopexie élective des cellules conjonctives de l'Escargot. Rev. suisse Zool. v. 49, p. 190-193.

1943 Elimination du pigment et de quelques colorants colloüdaux par la paroi intestinale du têtard. C. R. Soc. Phys. et Hist. nat. Genève, v. 60, p. 238-241.

1945 - Sur la fonction excrétrice transitoire de la cellule intestinale du têtard. Résumé dans Med. Wochensch. 75 Jahrg.

1906. Duesberg, G.: Contribution à l'étude des phénomènes histologiques de la métamorphose chez les amphibiens Anoures. Arch. Biol. v. 22, p. 163-228.

1904. Enriques, P.: Über pigmentierte Wanderzellen des Frosches. Anat. Anz. Bd. 24, S. 542-544.

1904. Gaupp, E.: Anatomie des Frosches. Braunschweig 1904.

1932a. Gérard, P. et R. Corder.: Etudes histophysiologiques sur le rein des anoures. Arch. biol. v. 43, p. 367-413.

1932b. - Sur l'élimination rénale d'encres de Chine de dispersions différentes. C. R. Assoc. Anat. 27e Réunion Nancy, p. 1-5.

1933. _ Sur le rapport existant entre maximum d'athrocytose et dimensions des particules résorbées dans les néphrons ouverts. C. R. Soc. Biol. v. 115, p. 198-201.

1933. _ Recherches histophysiologiques sur le pro-et le mésonéphros des têtards d'anoures. C. R. Assoc. Anat. 28e Réunion, Lisbonne, 33, p. 1-11.

1943. Gineste, P. J.: Recherches sur le pigment noir du foie des Amphibiens. C. R. Soc. Biol. v. 137, p. 25.

1924. Herzog, Fr.: Endothelien der Froschzunge als Phagocyten und Wanderzellen. Zeitsch. exper. Med. Bd. 43. Cité d'après Schmidt 1931.

1926. Huppert, M.: Beobachtungen am Magen und Darmkanal des Frosches bei Verfütterung oder Injektion von Farbstoffen. Z. Zellforsch. Bd, 3. S. 602-614.

1934. Janes, R. G.: Studies on the Amphibian digestive systeme 1. Hislogical changes in the alimentary tract of anuran Larvae during involution.J. exp. zool. v. 67, p. 73-92. 
1923. Krause, R.: Mikroskopische Anatomie der Wirbeltieren in Einzeldarstellungen, 1921-1923.

Cité d'après Patzelt, V.: Der Darm. Handb. d. mikr. Anat. des Menschen Bb V/3 1936, p. 201.

1927. Maximow, A.: Embryonale Entwicklung des Blutes. 4 Amphibien Handb. d. mik. Anat. des Menschen 11/1, p. 523.

1918. Moellennorff, W. v.: Zur Morphologie der vitalen Granulafärbung. Arch. f. mik. Bd. 90, S. 463-502.

1919. _ Über Funktionsbeginn und Funktionsbestimmung in der Harnorganen von Kaulquappen. Sitzungsber. Heidelb. Akad. d. Wissensch. Jahrgang 1919. p. 3-26.

1925. — Beiträge zur Kenntnis der Stoffwanderungen bei wachsenden Organismen. Zeitsch. Zellf. u. mik. Anat. Bd. 2. p. 129-202.

1909. Prenant, A.: Observations sur les cellules pigmentaires et sur le pigment des amphibiens. C. R. Assoc. Anat. 27e Réunion, Nancy 1909, p. 44-60.

1931. Sснмпт, G.: Die vitale Trypanblauspeicherung bei Salamanderlarven. .Ztschr.f. Anat. u. Entwicklungesch. Bd. 96, p. 68-83. 


\section{$2 \mathrm{BHL}$ Biodiversity Heritage Library}

Du Bois, Anne Marie. 1946. "Athrocytose et transfert des colorants colloïdaux et du pigment chez l'embryon et le têtard de grenouille." Revue suisse de zoologie 53, 1-31. https://doi.org/10.5962/bhl.part.117712.

View This Item Online: https://www.biodiversitylibrary.org/item/148532

DOI: https://doi.org/10.5962/bhl.part.117712

Permalink: https://www.biodiversitylibrary.org/partpdf/117712

\section{Holding Institution}

American Museum of Natural History Library

\section{Sponsored by}

BHL-SIL-FEDLINK

\section{Copyright \& Reuse}

Copyright Status: In copyright. Digitized with the permission of the rights holder.

Rights Holder: Muséum d'histoire naturelle - Ville de Genève

This document was created from content at the Biodiversity Heritage Library, the world's largest open access digital library for biodiversity literature and archives. Visit BHL at https://www.biodiversitylibrary.org. 